\title{
Deeply Virtual Exclusive Reactions with CLAS
}

\author{
Valery Kubarovsky* \\ Jefferson Laboratory, Newport News, VA, USA \\ For the CLAS Collaboration \\ E-mail: vpk@jlab.org
}

Deeply virtual exclusive reactions offer an unique opportunity to study the structure of the nucleon at the parton level as one has access to Bjorken $x_{B}$ and momentum transfer to the nucleon $t$ at the same time. Such processes can reveal much more information about the structure of the nucleon than either inclusive electroproduction or elastic form factors alone. Dedicated experiments to study Deeply Virtual Compton Scattering (DVCS) and Deeply Virtual Meson Production (DVMP) have been carried out in Hall B at Jefferson Lab. DVCS helicity-dependent and helicityindependent cross sections and beam spin asymmetries have been measured with CLAS, as well as cross sections and asymmetries for the $\pi^{0}, \eta, \rho^{0}, \rho^{+}, \omega$ and $\phi$ for exclusive electroproduction. The data were taken in a wide kinematic range in $Q^{2}=1-4.5 \mathrm{GeV}^{2}, x_{B}=0.1-0.5$, and $|t|$ up to 2 $\mathrm{GeV}^{2}$. We will discuss the interpretation of these data in terms of traditional Regge and Generalized Parton Distributions models. We view the work presented in this report as leading into the program of the Jefferson Lab $12 \mathrm{GeV}$ upgrade. The increased energy and luminosity will allow us to acquire data at much higher $Q^{2}$ and $x_{B}$, and perform Rosenbluth L/T separations of the cross sections.

35th International Conference of High Energy Physics - ICHEP2010,

July 22-28, 2010

Paris France

${ }^{*}$ Speaker. 


\section{Introduction}

The mapping of the nucleon's structure in terms of the Generalized Parton Distributions (GPDs) is one of the major objectives of Jefferson Lab. The GPDs give access to the complex internal structure of the nucleon, such as correlations between the parton transverse spatial and longitudinal momentum distributions. They provide a unified picture of the nucleon form factors, polarized and unpolarized parton distributions, and provide access to the contribution of the total parton angular momentum to the nucleon spin.

Hard exclusive processes of a meson or photon electroproduction of the nucleon are the key processes to access the GPDs from the experimental observables. At high photon virtuality $Q^{2}$ and high energy transfer $v$ in the Bjorken scaling regime, the scattering amplitude of the hard exclusive processes factorizes into a hard scattering part (exactly calculable in QCD) and a nucleon structure part described by the GPDs.

\section{Deeply virtual Compton scattering}

CLAS has published the DVCS beam-spin asymmetries (BSA) [1, 2] and longitudinally polarized target asymmetries [3]. By fitting these data in a largely model-independent way, the imaginary parts of two Compton Form Factors $\operatorname{Im}(\tilde{\mathscr{H}})$ and $\operatorname{Im}(\mathscr{H})$ were extracted with uncertainties on the order of 30\% [4]. In the framework of the dominance of the Generalized Parton Distribution $H$ and twist-2 accuracy the real and imaginary parts of the Compton Form Factor $\mathscr{H}$ were extracted [5]. In addition to the CLAS data, helicity-independent and helicity-dependent DVCS cross sections were used in this analysis [6]. These are the first attempts to get access to the GPDs from experimental data. The CLAS group is now working on the determination of the absolute DVCS cross sections in a wide kinematic region.

\section{Pseudoscalar mesons $\pi^{0}$ and $\eta$ electroproduction}

The reactions $e p \rightarrow e^{\prime} p^{\prime} \pi^{0}$ and $e p \rightarrow e^{\prime} p^{\prime} \eta$ were measured with the CLAS spectrometer at a beam energy of about $6 \mathrm{GeV}$. The virtual photon cross section can be written as

$$
\frac{d \sigma}{d \Omega_{\pi}}=\sigma_{T}+\varepsilon \sigma_{L}+\varepsilon \sigma_{T T} \cos 2 \phi+\sqrt{2 \varepsilon(1+\varepsilon)} \sigma_{L T} \cos \phi+h \sqrt{2 \varepsilon(1-\varepsilon)} \sigma_{L T}^{\prime} \sin \phi,
$$

where $\phi$ denotes the azimuthal angle between the hadronic and leptonic scattering planes and $h$ is the electron beam polarization.

The large acceptance of CLAS enabled the data to be grouped into about 2000 bins in $Q^{2}, t x_{B}$ and $\phi$ for $\pi^{0}$ and about 1000 bins for $\eta$ production. For unpolarized electrons $(h=0)$ the separation of the $\phi$ dependence in moments of a constant, $\cos \phi$, and $\cos 2 \phi$ allows us to obtain $\sigma_{T}+\varepsilon \sigma_{L}, \sigma_{T T}$ and $\sigma_{L T}$ separately.

\section{Separated structure functions}

The separated structure functions $\sigma_{T}+\varepsilon \sigma_{L}, \sigma_{T T}$, and $\sigma_{L T}$ as a function of $-t$ were obtained from fits to the differential cross section data for 15 intervals in $Q^{2}$ and $x_{B}$, two of which are 
shown in Fig 1. Since the structure functions at the present kinematics cannot be described by the leading order handbag approach, we display the results of a Regge-type analysis [7]. It appears that the Regge approach can account for the main features of the structure functions, both in their magnitude and their relationships to each other.
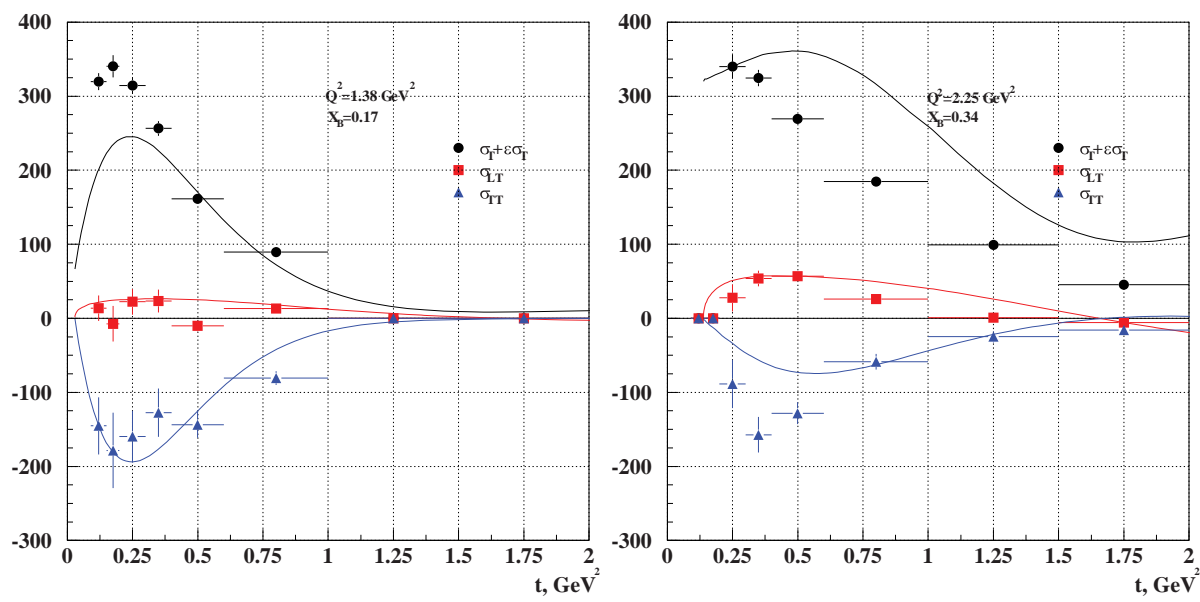

Figure 1: The separated structure functions $\sigma_{T}+\varepsilon \sigma_{L}, \sigma_{T T}$ and $\sigma_{L T}$ as a function of $-t$ at $\left(Q^{2}=1.38 \mathrm{GeV}^{2}\right.$, $\left.x_{B}=0.17\right)$ and $\left(Q^{2}=2.25 \mathrm{GeV}^{2}, x_{B}=0.34\right)$ obtained with the CLAS spectrometer. The curves are the results of the Regge-based calculation of Ref. [7]

\section{$t$-slope}

In the Regge framework the cross sections can be expressed according to the following form: $d \sigma / d t \propto \exp \left(B\left(x_{B}\right) t\right)$ with slope $B\left(x_{B}\right)=2 \alpha \ln \left(1 / x_{B}\right)$, where the parameter $\alpha \sim 1.1$. Remarkably, the shape of the slope is seen to be almost completely independent of $Q^{2}$ [8]. Equally remarkably, it follows the Regge predicted shape with $\alpha \sim 1.1$ with no further adjustable parameters.

\section{The ratio of cross sections for $\eta$ and $\pi^{0}$}

Even though the current experiments are limited in $Q^{2}$ and $t$, it has been argued [9] that precocious factorization of the ratios of cross sections as a function of $x_{B}$ could be valid at relatively lower $Q^{2}$ than for the cross sections themselves. The ratio of cross sections for $\pi^{0}$ and $\eta$ electroproduction from our data [8] is almost independent of $x_{B}$ and varies from 0.3 to 0.4 with increasing t. This is in contrast to the prediction [9], where this ratio is equal to $\sim 1$. However, we cannot compare directly with [9] since $\sigma_{L}$ and $\sigma_{T}$ were not separated.

\section{Beam spin asymmetry}

The beam spin asymmetry (BSA) directly yields the $L-T$ interference structure function $\sigma_{L T}^{\prime}$. Any observation of a non-zero BSA would be indicative of an L-T interference. Sizable beam spin asymmetries for exclusive $\pi^{0}$ and $\eta$ meson electroproduction have been measured above the resonance region over a large number of kinematic bins for the first time $[10,11]$. 
The large non-zero asymmetries imply that both transverse and longitudinal amplitudes participate in the process. The results of a Regge model calculation at least qualitatively describe the experimental data.

\section{Vector mesons electroproduction}

The CLAS collaboration has already published the cross sections for the $\rho^{0}[12], \omega[13]$ and $\phi$ [14]. For the $\rho^{+}$channel, the first-ever measurement of its cross section was recently published [15].

Fig. 2 shows the total longitudinal cross section $\sigma_{L}\left(\gamma^{*} p \rightarrow p \rho^{0}\right)$ as a function of $W$ for fixed $Q^{2}$. The JML model [16] (the dot-dashed curve) is able to successfully reproduce the cross sections for almost all of our $\left(Q^{2}, W\right)$ range.

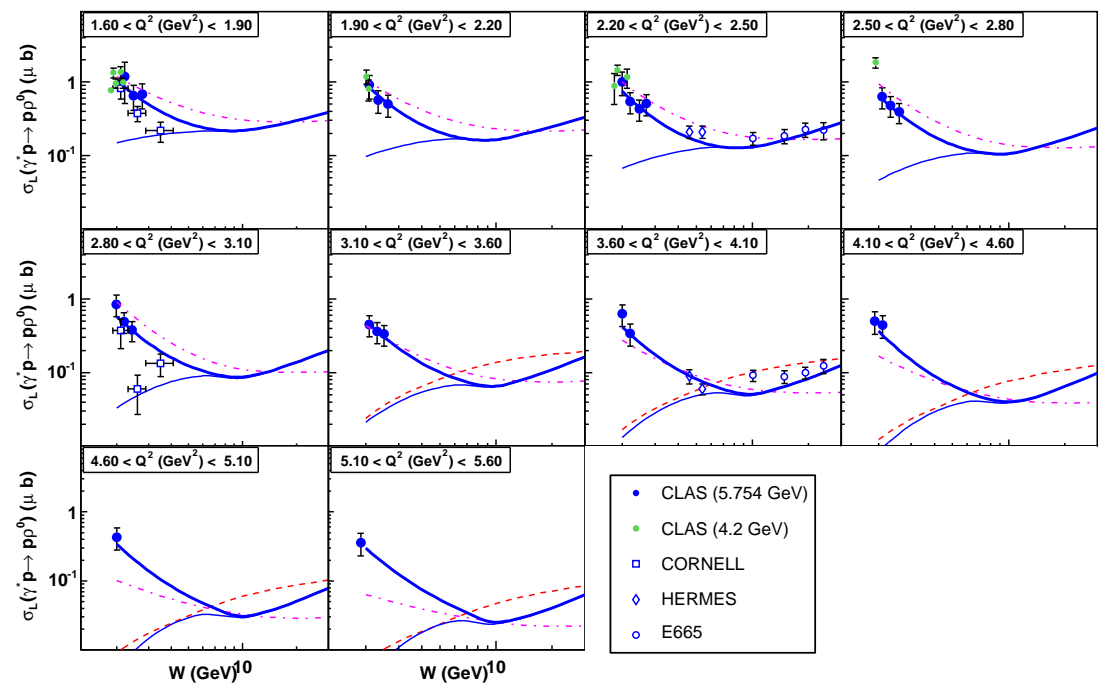

Figure 2: Longitudinal cross section as a function of $W$ at fixed $Q^{2}$ for the reaction $\gamma^{*} p \rightarrow p \rho^{0}$. The dashed curve shows the result of the GK calculation [17] and the thin solid curve shows the result of the VGG calculation [18]. The thick solid curve is the VGG calculation with the addition of the new $t$-channel meson exchange term. The dot-dashed curve shows the results of the Regge JML calculation.

In Fig. 2, the dashed line shows the results of the GK model [17], while the thin solid line shows the result of the VGG model [18]. We see that GPD models give a good description of the high and intermediate $W$ region, down to $W \sim 5 \mathrm{GeV}$. At high $W$, the slow rise of the cross section is due to the gluon and sea contributions, while the valence quarks contribute only at small $W$. At lower $W$ values, where the new CLAS data lie, both the GK and VGG models fail to reproduce the data. This discrepancy can reach an order of magnitude at the lowest $W$ values. The trend of these particular GPD calculations is to decrease as $W$ decreases, whereas the data increase. The same behavior was observed in the low $W$ region for the exclusive electroproduction of the $\rho^{+}$. An attempt to reconcile the GPD calculation with the low $W \rho^{0}$ cross sections is presented in Ref.[19]. Through a toy-model, $t$-channel meson exchanges are included in the GPDs, and the result of this calculation (actually a fit) is illustrated by the thick blue curve in Fig. 2. 


\section{Conclusion}

Cross sections and asymmetries for the $\gamma, \pi^{0}, \eta, \rho^{0}, \rho^{+}, \omega$ and $\phi$ exclusive electroproduction in a wide kinematic range of $Q^{2}, t$ and $x_{B}$ have been measured and initial analyses already are showing remarkable results. We view the work presented here as leading into the program of the Jefferson Lab $12 \mathrm{GeV}$ upgrade. The increased energy and luminosity will allow us to extend the analysis presented here at much higher $Q^{2}$ and $x_{B}$, as well as to perform Rosenbluth $L / T$ separations.

We acknowledge the outstanding efforts of the staff of the Accelerator and Physics Divisions at Jefferson Lab that made this experiment possible. We also acknowledge useful discussions with the DVCS CLAS group. This work was supported by the U.S. Department of Energy and National Science Foundation. Jefferson Science Associates (JSA) operates the Thomas Jefferson National Accelerator Facility for the United States Department of Energy under contract DE-AC0506 OR23177.

\section{References}

[1] S. Stepanyan, et al., Phys. Rev. Lett. 87:182002 (2001)

[2] F.-X. Girod, et al., Phys. Rev. Lett. 100 :162002 (2008)

[3] S. Chen, et al., Phys. Rev. Lett. 97:072002 (2006).

[4] M. Guidal, Phys. Lett. B 689 ,156 (2010)

[5] H. Moutarde, Phys. Rev. D 79:094021 (2009).

[6] C. Munoz Camacho et al., Phys. Rev. Lett. 97:262002 (2006).

[7] J-M. Laget, [1004.1949] (2010).

[8] V. Kubarovsky et al., arXiv:0802.1678 [hep-ex], 2007.

[9] M. I. Eides, L. L. Frankfurt and M. I. Strikman, Phys. Rev. D 59, 114025 (1999).

[10] R. De Masi et al., Phys. Rev. C 77:042201 (2008) [0711.4736].

[11] K. Joo et al., AIP Conf.Proc.915:607-610 (2007), to be published.

[12] S. Morrow et al., Eur. Phys. J. A 39, (2009) 5.

[13] L. Morand et al., Eur. Phys. J. A 24 (2005) 445.

[14] J. P. Santoro et al., Phys. Rev. C 78 (2008) 025210.

[15] A. Fradi, arXiv:1010.1198 [hep-ex], arXiv:1009.3775 [hep-ex], 2010.

[16] J.-M. Laget, Phys. Lett. B 489 (2000) 313;

F. Cano and J.-M. Laget, Phys. Rev. D 65 (2002) 074022;

J. M. Laget, Phys. Rev. D 70 (2004) 054023;

F. Cano and J. M. Laget, Phys. Lett. B 551 (2003) 317.

[17] S.V. Goloskokov and P. Kroll, Eur. Phys. J.C 42 (2005) 281; Eur.Phys. J.C 50 (2007) 829.

[18] M. Vanderhaeghen, P.A.M. Guichon, M. Guidal, Phys. Rev. D 60, 094017 (1999).

[19] M. Guidal and S. Morrow, arXiv:0711.3743 (hep-ph). 